\title{
La competencia de internacionalización del profesorado universitario. Un reto para la educación superior
}

\section{The internationalization competence of university teachers. A challenge for higher education}

\author{
Beatriz Cortina-Pérez \\ Universidad de Granada \\ bcortina@ugr.es \\ http://orcid.org/0000-0003-2716-1333 \\ Antonio Medina Rivilla \\ Universidad Nacional de Educación a Distancia \\ amedina@edu.uned.es \\ https://orcid.org/0000-0002-1483-0668
}

Fechas · Dates

Recibido: 2019-01-10

Aceptado: 2019-11-20

Publicado: 2019-12-31
Cómo citar este trabajo · How to Cite this Paper

Cortina-Pérez, B., \& Medina, A. (2019). La competencia de internacionalización del profesorado universitario. Un reto para la educación superior. Publicaciones, 49(5), 209-224. doi:10.30827/publicaciones.v49i5.11228 


\title{
Resumen
}

La Universidad está llamada a satisfacer las demandas de la sociedad, así como a adaptarse a los cambios que en ella suceden. Conscientes de este hecho y del carácter global e internacional de la sociedad, las instituciones de Educación Superior españolas se han lanzado hacia la carrera de la internacionalización. Sin embargo, informes como los de la $O C D E$, entre otros, revelan el escaso efecto de estas políticas, dejando a España en una posición desfavorecida frente a otras instituciones internacionales. Asumiendo un desfase importante entre las demandas sociales y la formación del profesorado universitario, este artículo reflexiona sobre el papel de la la función docente en la internacionalización de las universidades y reclama la inserción en los decálogos de competencias del docente universitario de la competencia de internacionalización para que el profesorado sea capaz de afrontar este reto, y por ende, contribuir a la verdadera internacionalización de la Educación Superior. Tras hacer una revisión del papel internacional de la Educación Superior, analizamos desde esta perspectiva las funciones del profesor universitario, así como los modelos más conocidos de competencias docentes universitarias. Por último, proponemos una nueva competencia transversal de internacionalización del profesorado universitario, compuesta por dos subcompetencias que interaccionan con el resto de competencias docentes: la competencia comunicativa académica internacionalizadora (CCAI) y la competencia intercultural académica (CIA).

Palabras clave: Internacionalización; Educación Superior; Espacio Europeo de Educación Superior (EEES); competencia de internacionalización; formación del profesorado

\begin{abstract}
The university is expected to satisfy the demands of the society, as well as to adapt itself to its produced changes. Aware of this fact, and of the global and international character of the society, Spanish HEIs have launched themselves into internationalization. However, reports such as those by the OECD, among others, reveal the poor effect of these policies, leaving Spain to an unprivileged position compared to other European institutions. Detecting an important gap between social demands and university teacher training programmes, this paper reflects on the role of the academics' functions within the internationalization of universities and claims the introduction of the internationalization competence in the university teachers' competence lists so as to help teachers face this challenge, therefore, contributing to the real internationalization of $\mathrm{HE}$. After reviewing the role of internationalization within $\mathrm{HE}$, we analyze from international perspective academics' duties, as well as accepted teaching competence taxonomies for university teachers. Finally, we propose the unlisted transversal competence of internationalization for academics, being compounded of two subcompetences that interact with all the teaching competences: the international academic communicative competence (IACC), and academic intercultural competence (AIC).
\end{abstract}

Keywords: Internationalization; Higher Education; European Area of Higher Education (EAHE); internationalization competence; university teachers' training

"Europa no se salva si no entra de nuevo en caja, y este encaje tiene que ser más riguroso que los que hasta ahora usados y abusados. Nadie deberá escapar a él. Tampoco el hombre de la ciencia"

(Ortega y Gasset, 1930). 


\section{Introducción}

La universidad, tal y como la defendía ya Unamuno al albor del siglo XX, debe afrontar las demandas sociales de cada tiempo, haciendo uso del progreso y del conocimiento con este fin. Consecuentemente, los centros de Educación Superior "deben estar atentos a las necesidades de dicha sociedad, no vivir de espaldas a ellas y por lo tanto deben ser innovadoras en sus tareas y en su organización" (Ortega, 2002, p. 68). La sociedad del siglo XXI, influenciada en parte por la eclosión tecnológica, se caracteriza precisamente por su globalización y su dinamismo; lo que ahora está de moda, en breve no, y así sucesivamente, trazando una espiral de cambio incesante. Como ya apuntaba Lyotard (1994), en esta nueva sociedad se rompen las barreras espacio-tiempo para construir una civilización global que no se limita a un único lugar y a una relación de diacronía, sino que interactúa en múltiples contextos y de forma sincrónica. En un breve periodo de tiempo hemos pasado de la sociedad de la comunicación a la sociedad de la información, donde el conocimiento se construye y se transforma, no es estático; en el mundo actual, no basta con estar informado, sino que se demanda la difusión y la interacción con otros sujetos sobre el conocimiento adquirido a fin de contrastarlo, compararlo y, si fuera necesario, ampliarlo o refutarlo. En esta misma línea globalizadora, Esteban y Menjívar (2011) sugieren que "en el marco de la mundialización, son claros los cambios sociales que ha traído la sociedad de la información, que ha convertido a las sociedades cerradas en sociedades abiertas a la llamada aldea global" (p. 23).

La universidad, con otra cadencia pero con el mismo espíritu regenerador, intenta transformarse al ritmo que impone la sociedad. De ahí que en la última década hayamos asistido a un profundo cambio de la Educación Superior, que busca la promoción de la internacionalización en todos sus ámbitos de actuación (Harris, 2008; Kahn \& Agnew, 2017; Lasagabaster, Cots \& Palma, 2011; Mancho-Barés, 2013; Torres, Badillo, Valentín \& Ramírez, 2015). En concreto, en el caso de España, las políticas educativas europeas han contribuido considerablemente a este cambio. Como indican Fernández y Gijón (2011), "la universidad no ha sido siempre la misma institución y los cambios que se producen en ella determinan cambios en la profesión académica y la consecuente profesionalización de sus docentes" (p. 94). Pero la complejidad de este fenómeno reside en el hecho de que conseguir una verdadera internacionalización pasa, no solo por adoptar medidas que fomenten el libre intercambio, sino que se forme a los agentes implicados para que puedan hacer un uso eficiente de estas cooperaciones y de esta dimensión internacional de la Educación Superior.

Este artículo defiende la necesidad de integrar la internacionalización dentro de las competencias del profesorado universitario para conseguir una verdadera proyección internacional de la Universidad puesto que, como acabamos de subrayar, el profesorado es la verdadera alma de la transformación universitaria y, si no se le forma para ello, no será capaz de afrontar estos nuevos retos con eficacia.

\section{La formación de un nuevo espacio internacional de Educación Superior}

El acuerdo de Schengen de 1985 marcó un hito importante en el desarrollo de la internacionalización del sistema educativo europeo. La finalización de controles fronterizos entre los Estados miembros de la Unión Europea tras su entrada en vigor a mitad de 
los años 90 facilitó la libre movilidad de docentes y discentes y, con ello, el fomento de políticas con vocación internacionalizadora. De forma paralela, el Plan de Acción de la Comunidad Europea para la Movilidad de Estudiantes Universitarios (European Community Action Scheme for the Mobility of University Students), más conocido como Programa ERASMUS, fue tomando forma. Su objetivo fundamental era favorecer la movilidad, así como la dimensión europea de la enseñanza superior. Con estas y otras medidas, en los años 90 la acción internacionalizadora se convirtió en una prioridad en materia de política europea universitaria (Barrow, Didou \& Mallea, 2003). Como consecuencia, asistimos a la popular Declaración de Bolonia que claramente apostaba por la promoción de la movilidad de estudiantes, profesores y personal de administración y servicios de las instituciones de Educación Superior, así como enfatizaba la dimensión europea en el desarrollo integrado de planes de estudio para la formación e investigación y la cooperación entre instituciones (Consejo de Europa, 1999). En este proceso tuvo una especial relevancia la implantación del sistema de créditos ECTS y Suplemento Europeo al Título (SET), dado que se consiguieron establecer mecanismos de equiparación entre los estudios, entre las evaluaciones, etc. de distintas universidades europeas y, con ello, se ha facilitado el intercambio humano y de conocimiento. Se genera el conocido Espacio Europeo de Educación Superior que pretende vigorizar un área europea de educación terciaria competitiva a nivel internacional. Cada país miembro de este espacio común debe contribuir con políticas propias para el objetivo final de la UE que es la modernización del sistema universitario europeo (Bergan \& Rauhvargers, 2006).

En el ámbito más cercano, la LOU -Ley Orgánica de Universidades- (Ministerio de Educación, 2007), y su posterior desarrollo y modificación, ha sido el cambio legislativo que Europa demandaba a la Educación Superior española para su modernización e internacionalización. Además, el Gobierno de España generó en el 2009 el plan de actuación Estrategia Universidad 2015 -EU2015- con el objetivo de conseguir la actualización de la universidad española y, por ende, convertirla en una universidad competitiva a nivel internacional.

La Estrategia Universidad 2015 representa una acción integral y pone en primer plano un conjunto de acciones ordenadas por ejes creativos en cuatro ámbitos cuyo desarrollo conjunto, armónico y coordinado debería permitir dar un salto importante en su competitividad individual y colectiva en el conjunto de sistemas universitarios de excelencia en el mundo. (Ministerio de Educación, 2009, p. 10).

Las políticas internacionales en el contexto español se dirigen fundamentalmente a los siguientes aspectos (Ministerio de Educación, 2009):

1. la oferta universitaria;

2. los recursos humanos de los que dispone;

3. el incremento del atractivo de las universidades españolas desde el extranjero; y

4. la dimensión social global.

Como deja patente este documento, el Gobierno Español considera un objetivo cardinal la internacionalización de las instituciones de Educación Superior. Es tal la importancia para las instituciones de este componente internacional en la universidad que el siguiente paso fue la generación de la "Estrategia para la Internacionalización 
de las universidades 2015-2020" (Ministerio de Educación, 2016) que se propone los siguientes objetivos:

1. consolidar el sistema universitario altamente internacionalizado

2. aumentar el atractivo internacional de las universidades

3. promover la competitividad internacional del entorno

4. intensificar la cooperación en educación superior con otras regiones del mundo

Sin embargo, y a pesar de los esfuerzos y predisposición de los dirigentes, la Universidad española aún está en un proceso de consecución de un perfil claramente internacional.

\section{El proceso de internacionalización del sistema universitario español}

La internacionalización en el contexto de la Educación Superior es la integración del componente internacional o intercultural en la vida académica al servicio de la institución (Knight, 1999), con el objeto de aumentar su potencial docente, de investigación y de gestión (De Wit, 2020). Podemos afirmar que la universidad posee de forma innata esta dimensión que busca la cooperación entre universidades sin atender a razones de situación geográfica (Haug, 2010). Gracias a este énfasis internacional, la Educación Superior consigue modernizarse, promover la competitividad en el sector económico y tecnológico, lograr estándares académicos internacionales de calidad de la enseñanza y de la investigación, así como promover la diversidad cultural en las instituciones (Gijón \& Crisol, 2012; Montes \& Suárez, 2016). Además, esta dimensión está llamada a repercutir sobre el entorno en el que la universidad se sitúa (Ministerio de Educación, 2016), contribuyendo así a la integración de la universidad en la sociedad en la que se enmarca.

En el momento en el que buscamos que nuestros egresados sean competentes en un mercado laboral competitivo y dinámico, la internacionalización es una necesidad incuestionable y uno de los principales cambios que la Educación Superior ha experimentado (Bozu \& Imbernón, 2016; DeWitt, 2017). Como específica el documento Estrategia Universidad 2015:

La globalización del empleo y la internacionalización de los nuevos puestos de trabajo derivados de la incorporación de nuestro país en la Sociedad basada en el Conocimiento ha provocado que en el momento de adecuación de nuestros estudios al EEES se consideraran de forma especial la adquisición de conocimientos, habilidades y competencias de internacionalización con el fin de preparar a nuestros futuros egresados para un mundo cada vez más globalizado a nivel del empleo. (Ministerio de Educación, 2009, p. 3)

Haug (2010) indica que la internacionalización de las universidades europeas ha pasado por tres fases: el fomento de la movilidad, la internacionalización de los estudios y, por último, de la institución, en concreto, en dos aspectos, la vida del campus y el uso de la experiencia internacional como acicate para la transformación; y precisamente, es en esta última fase donde más han fallado las estrategias de internacionalización. 
Según el propio autor: "Estas posibilidades son poco utilizadas en muchas universidades, incluso en España, donde, a pesar de una fuerte participación en programas de intercambio, los campus no "huelen" mucho a internacional" (Haug, 2010, p. 25).

Aunque atraemos a un gran número de estudiantes internacionales a nuestro país, principalmente Erasmus, no son tantos los alumnos extranjeros fuera de estos programas que escogen España para realizar sus estudios universitarios de grado y posgrado de acuerdo con el informe de la OCDE (Ministerio de Educación, Cultura y Deporte, 2013) o el de Eurydice de 2012 (Ministerio de Educación, Cultura y Deporte, 2013b). El porcentaje de alumnos de posgrado en nuestro país, uno de los criterios de calidad de la Educación Superior, es de 3.2\% frente al 6.9\% de la OCDE (Ministerio de Educación, Cultura y Deporte, 2013). De manera más reciente, el informe Eurydice sobre movilidad académica en el EEES (Comisión Europea, 2017), Ilama la atención sobre la necesidad de una internacionalización más profunda de la Educación Superior en lo referente al profesorado: "Si bien el personal académico tiene la gran responsabilidad de llevar adelante estos objetivos, tienden a mencionarse explícitamente solo en relación con la movilidad" (Comisión Europea, 2017, p. 12).

En el ámbito de la producción científica, uno de los ranking de universidades más prestigiosos a nivel internacional, el Academic Ranking of World Universities (ARWU) de la Universidad de Shanghái, deja fuera a las universidades españolas del conjunto de las 200 mejores en el 2017. Según indica la Comisión de Expertos para la Reforma del Sistema Universitario Español:

[...] aunque el lugar de una universidad determinada siempre puede discutirse, no cabe cuestionar el paisaje general que muestran [los rankings internacionales] de nuestras universidades: si bien hay centros excelentes, ninguna universidad española juega en la primera división universitaria. Y aunque la financiación que reciben es claramente insuficiente [...], ello no basta para justificar la situación actual. (Ministerio de Educación, 2013, p. 7).

Aunque esta afirmación incite al debate sobre la forma de medir la calidad de las universidades, la realidad es que la Universidad española, en el momento actual, no es competitiva a nivel internacional. El desembolso del Gobierno español en profesores y alumnos supera la media de los países de la OCDE (Ministerio de Educación, Cultura y Deporte, 2013) y, sin embargo, estamos muy por debajo en producción científica. Recientemente, en un ciclo de conferencias titulado "Los cambios que precisa la educación en España" ${ }^{\prime \prime}$, Salvador García-Atance apuntaba que la razón no debía buscarse en la cantidad de inversión realizada, sino en la gestión de ésta.

A su vez, el informe "Temas candentes de la universidad española", elaborado por PricewaterhouseCoopers S.L. en colaboración con la Fundación Europea Sociedad y Educación y de Banco Santander en 2013, resalta la escasa internacionalización de la universidad española y señala como una de las salidas a esta situación, la contratación de profesores de excelencia internacionales, al tiempo que se reconoce que uno de los principales impedimentos para ello es la falta de competitividad de los salarios. En esta captación de cerebros extranjeros, también incide la Estrategia 2015-2020 (Ministerio de Educación, 2016).

A la vista de todos estos informes, queda de manifiesto que una mayor internacionalización contribuiría muy positivamente a mejorar el sistema educativo superior

$1 \mathrm{http}: / /$ www.fundacionmutua.es/FundMM/conferenciaAudio.jsp 
español (De Wit, 2020). Se están tomando medidas prioritariamente centradas en la internacionalización del alumnado para que consigamos unos egresados internacionalmente competentes, por ejemplo, incentivando la formación y la acreditación en las lenguas extranjeras, o fomentando la obtención del Suplemento Europeo al Título (SET). No obstante, se está obviando una de las piezas clave del entramado universitario: el profesorado. No debemos olvidar que, en muchas ocasiones, es el docente universitario el que gestiona o facilita acuerdos internacionales o el que participa y trabaja por la consolidación de redes internacionales que luego dan sus frutos en proyectos internacionales o titulaciones conjuntas (Comisión Europea, 2016). Aunque el número total de movilidades del docente universitario va creciendo, aún no es suficiente y es que muchos de los profesionales achacan su escasa proyección internacional a su escaso dominio de otras lenguas, principalmente del inglés (Jato, Muñoz \& García, 2014). Por otra parte, el profesor universitario es un referente para el estudiante, es un modelo profesional en el que reflejarse, por lo que una internacionalización mayor del profesorado universitario redundaría en una intensificación de la internacionalización de los estudiantes universitarios (Morales \& Cabrera, 2012; Monereo, 2013).

En lugar de potenciar la atracción de profesores extranjeros, tal y como sugiere el informe de expertos del Ministerio o la OCDE, ¿no tendría más lógica utilizar los recursos humanos con los que la universidad española cuenta? Se trata de poner la solución en la raíz del problema; se trata, como no podía ser de otra forma en la Educación Superior actual, de formación en competencias.

Mas Torelló (2011) propone que el camino para conseguir una verdadera reforma educativa en el sector terciario pasa irremediablemente por otorgar al profesorado y al logro de sus competencias la importancia real que tienen. Como recuerda Zabalza (2012), “Los profesionales que ejercemos esa función [profesor universitario] debemos estar en posesión de aquel conjunto de competencias que permitirá que lo hagamos dignamente" (p. 13). Por ello, creemos necesario una revisión de las competencias docentes actuales a fin de poder plasmar un modelo que realmente haga frente a las necesidades que demanda la sociedad actual y, por ende, la educación terciaria.

\section{Análisis de las competencias profesionales desde la internacionalización}

Existe gran disparidad de opiniones sobre el constructo de competencia, principalmente motivado por la polisemia del término, lo que ha derivado en numerosos debates sobre el modelo de competencias del profesorado universitario. Entendemos competencia como "la síntesis global de capacidades, habilidades y estilos peculiares para atender realidades complejas y estar abierto a encontrar las soluciones más valiosas en las situaciones problemáticas" (Medina \& Domínguez, 2006, p. 69). Moreneo, Sánchez-Busqués y Suñé (2012) definen las competencias profesionales como "el conjunto integrado de conocimientos de distinta naturaleza [...] que permiten a su portador enfrentar con éxitos problemas prototípicos (frecuentes) y emergentes (del fututo próximo) vinculados a un determinado escenario" (p. 81). En este sentido, las competencias profesionales del profesorado universitario serán aquel conjunto de capacidades, habilidades y estilos peculiares que le permitan afrontar con éxito las tareas propias de su profesión en sus tres vertientes: la docente, la investigadora y la gestora. 
Existen múltiples estudios sobre competencias del profesorado universitario, sin embargo resulta laborioso encontrar modelos que desglosen estos tres ejes fundamentales de forma conjunta y que además incluyan esta dimensión internacional. No queremos convertir este artículo en una enumeración de modelos de competencias, pero creemos oportuno para el objeto de nuestra investigación analizar los compendios más relevantes desde la perspectiva de la internacionalización.

Uno de los modelos pioneros y más recurrente de competencias profesionales del profesorado universitario es el diseñado por Zabalza (2002) que alude a 10 competencias docentes:

1. Planificación del proceso enseñanza-aprendizaje

2. Selección y preparación los contenidos disciplinares

3. Competencia comunicativa didáctica

4. Manejo de las nuevas tecnologías

5. Diseño metodológico y organización de actividades

6. Comunicación y relación con los alumnos

7. Tutorización

8. Evaluación

9. Reflexión e investigación sobre la enseñanza

10. Identificación con la institución y el trabajo en equipo

Esta taxonomía se centra principalmente en la labor docente, dejando de lado los otros dos perfiles profesionales del profesor universitario: investigación y gestión. Aunque no incide directamente sobre la internacionalización, Zabalza (2002) sí reconoce, a través de la competencia institucional, la necesidad del profesorado de sentirse miembro de una comunidad universitaria y poder representarla en distintos contextos, sin excluir obviamente el ámbito internacional.

Por su parte, Valcárcel (2005) desglosa un modelo competencial del docente universitario en el contexto del EEES más amplio e incluye competencias tanto generales como específicas (tabla 1). A pesar de contener aspectos de los tres perfiles del profesor universitario, se encuentra desequilibrado a favor de la actividad docente. Valcárcel reivindica la socialización como forma de desarrollo profesional y, por consiguiente, la necesidad de traspasar fronteras nacionales para favorecer una comunidad universitaria internacional.

Tejada (2002) sintetiza la actividad docente en tres competencias claves para el profesor universitario:

1. Competencias teóricas o conceptuales que permitan al profesor integrar el saber y el saber hacer en su actuación docente.

2. Competencias psicopedagógicas y metodológicas que faciliten la aplicación del saber y del saber hacer en la enseñanza.

3. Competencias sociales, integrando el saber ser y saber estar a fin de mejorar la calidad de su actividad docente. 
Tabla 1

Resumen de competencias del docente universitario

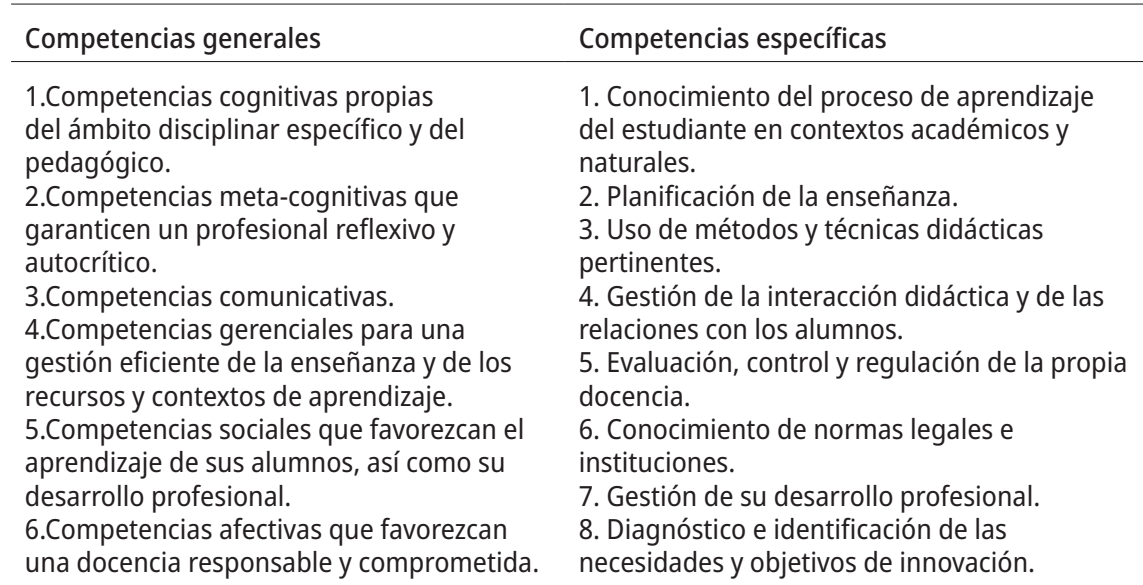

Fuente: Valcárcel (2005)

La formación en estas competencias resulta imprescindible, según Tejada (2009), para desempeñar la labor docente, pero además propone que "habría que asumir la internacionalización de la formación de formadores, tanto inicial como continua, ampliando los escenarios sociogeográficos de dicha formación" (p. 4). La defensa que hace el autor de la internacionalización está más bien enfocada hacia el contexto concreto de la formación, más que incluir de forma explícita la proyección internacionalizadora de la función docente en su modelo.

Por último, Medina, Domínguez y Ribeiro (2011) recopilan sucintamente los principales trabajos de competencias del profesorado universitario y establecen el siguiente decálogo de competencias profesionales:

- Innovación de la docencia

- Implicación en la sociedad del conocimiento

- Comunicativa, en cuanto a la interacción didáctica

- Planificación del conocimiento científico y de la docencia

- Desarrollo del sistema y estrategias metodológicas

- Valoración de las dimensiones europeas de la Educación Superior

- Tutorización

- Identidad profesional

- Diseño de medios y recursos

- Organización de los aprendizajes

- Evaluadora

Este listado incluye explícitamente la dimensión europea entre las competencias aludiendo a la conciencia de un espacio de Educación Superior que excede el ámbito local 
de cada universidad y donde los docentes tendrán que afrontar el reto de pertenecer a una comunidad educativa internacional.

En suma podemos decir que los modelos de competencias del profesorado están principalmente centrados en la actividad docente. Observamos que en la mayoría de clasificaciones que hemos analizado, y principalmente las más recientes, contemplan algún componente internacionalizador, sin embargo, este no es palpable de forma explícita en los distintos listados de competencias. Si queremos tener una verdadera proyección internacional de la universidad española debemos empezar por dotar de las competencias necesarias para ello al profesorado que la integra. Es por este motivo por el que creemos necesario incluir una competencia más en el desglose de competencias del profesorado universitario: la competencia de internacionalización.

\section{La competencia de internacionalización del profesorado universitario}

Para poder definir la competencia de internacionalización consideramos imprescindible realizar a priori un análisis de la función del profesorado universitario con proyección internacional. Tal y como apuntan diversos autores (De Ketele, 2003; Ruíz, Más, Tejada \& Navío, 2008), así como los documentos legales base -Artículo 65 de la Ley Orgánica de Universidades o el Estatuto del Personal Docente e Investigador-, el perfil del profesorado universitario se desarrolla en tres áreas o ejes profesionales: docencia, investigación y gestión. En la tabla 2 recogemos una revisión actualizada de las funciones del profesor universitario que nos sirva de cimiento para definir y desglosar la competencia de internacionalización.

Se desprende de este decálogo de funciones que la internacionalización está presente en todas las áreas de trabajo del profesor universitario. Todas estas funciones se aplican a la actividad del docente universitario en distintos momentos de su carrera académica; de ahí que consideramos que la competencia de internacionalización es una competencia transversal del docente universitario puesto que le capacita para afrontar retos, solucionar problemas, y comprender realidades que se le plantean en su situación laboral y que incide directamente sobre toda su actividad profesional docencia, investigación y gestión-.

Si tenemos en cuenta la definición de internacionalización y de competencia que hemos detallado a lo largo de este estudio, entendemos la competencia de internacionalización como el conjunto de capacidades, estilos y habilidades del profesor universitario que les permite afrontar la proyección internacional de su carrera profesional y, por ende, de la comunidad universitaria en la que se integra favoreciendo la cooperación sin atender a barreras geográficas. Consideramos que la competencia de internacionalización se divide a su vez en dos subcompetencias que armoniosamente se complementan para afrontar la dimensión internacional de la función docente (figura 1):

1. La Competencia Comunicativa Académica Internacionalizadora (CCAI) implica el conocimiento lingüístico y la capacidad para comunicarse en una lengua, principalmente el inglés, como lingua franca del ámbito profesional del profesorado universitario. Fue ya en la década de los 70 Dell Hymes quien reivindicó el papel del contexto en la competencia comunicativa, definiéndola como la capacidad que permite la interacción significativa entre hablantes de una misma comunidad lingüística (Hymes, 1972). Partiendo de este concepto, la CCAI se define 
Tabla 2

Análisis de las funciones del profesor universitario desde la perspectiva de la internacionalización

\begin{tabular}{|c|c|}
\hline $\begin{array}{l}\text { Perfil profesor } \\
\text { universitario }\end{array}$ & Funciones del profesorado \\
\hline Función docente & $\begin{array}{l}\text { Impartir docencia en inglés bien en programas bilingües del propio } \\
\text { centro o participando en intercambios docentes con universidades } \\
\text { extranjeras. } \\
\text { Mantenerse actualizado en las tendencias metodológicas de } \\
\text { Educación Superior a nivel internacional a través de la participación en } \\
\text { foros internacionales y de lectura de revistas internacionales de alto } \\
\text { prestigio. } \\
\text { Tutorizar a estudiantes internacionales. } \\
\text { Participar en grupos de trabajo docentes/jornadas de intercambios de } \\
\text { experiencias docentes/etc. En el ámbito internacional que propicien } \\
\text { acciones de formación/innovación docente conjunta. } \\
\text { Participar en comisiones de evaluación interuniversitarias (tribunales } \\
\text { de doctorados/tesinas/TFM/ etc. internacionales). } \\
\text { Dirigir trabajos académicos en marcos internacionales (tesis, tesinas, } \\
\text { maestrías, etc.). }\end{array}$ \\
\hline $\begin{array}{l}\text { Función } \\
\text { investigadora }\end{array}$ & $\begin{array}{l}\text { Participar en grupos de investigación internacionales. } \\
\text { Buscar asesoramiento de expertos internacionales en materia de } \\
\text { investigación. } \\
\text { Generar producción científica a nivel internacional en revistas de } \\
\text { prestigio o editoriales internacionales. } \\
\text { Participar en congresos/foros internacionales para difundir la } \\
\text { investigación. } \\
\text { Mantenerse actualizado en el conocimiento disciplinar a través de } \\
\text { la participación en foros internacionales y de lectura de revistas } \\
\text { internacionales de alto prestigio. }\end{array}$ \\
\hline Función gestora & $\begin{array}{l}\text { Dominar los procedimientos burocráticos y convocatorias para } \\
\text { la concesión de ayudas públicas de carácter internacional para la } \\
\text { docencia y/o investigación. } \\
\text { Participar y consolidar consorcios internacionales para elaborar } \\
\text { propuestas conjuntas de proyectos para la docencia y/o investigación, } \\
\text { así como la elaboración de planes de estudios internacionales/ } \\
\text { conjuntos. } \\
\text { Propiciar la firma de convenios internacionales que repercutan en la } \\
\text { mejora de la institución, la promoción de relaciones e intercambio } \\
\text { interdepartamental e interuniversitario. } \\
\text { Fomentar la organización de jornadas académicas, intercambios de } \\
\text { experiencias educativas, congresos, seminarios, etc. internacionales. } \\
\text { Aplicar sistemas de evaluación de la garantía de la calidad a nivel } \\
\text { internacional. }\end{array}$ \\
\hline
\end{tabular}

como la capacidad de uso de la lengua de comunidad científica para poder acometer las tareas comunicativas propias de la docencia, de la investigación y de la gestión en un contexto universitario internacional. Se trata por tanto, de una parte esencial de la competencia de internacionalización ya que se erige como la llave para el acceso a la comunidad internacional.

2. Por otra parte, la Competencia Intercultural Académica (CIA) facilita al profesor universitario las destrezas necesarias para afrontar la diversidad cultural pro- 
pia de un contexto internacional. De acuerdo con Aneas (2003), la competencia intercultural comprende "aquellos conocimientos, habilidades y actitudes que permiten [...] negociar, comunicarse y trabajar en equipos interculturales y hacer frente a las incidencias que surgen en la empresa multicultural" (p. 305). Se trata, por tanto, de un aspecto primordial en la dimensión internacional de la función docente dado que responde al carácter intrínsecamente diverso de la sociedad actual. La CIA permitirá al docente afrontar con éxito situaciones culturalmente distintas a través del conocimiento y la comprensión de otras culturas académicas -sensibilidad intercultural (Bennet, 1986; Hammer, 1999)-.

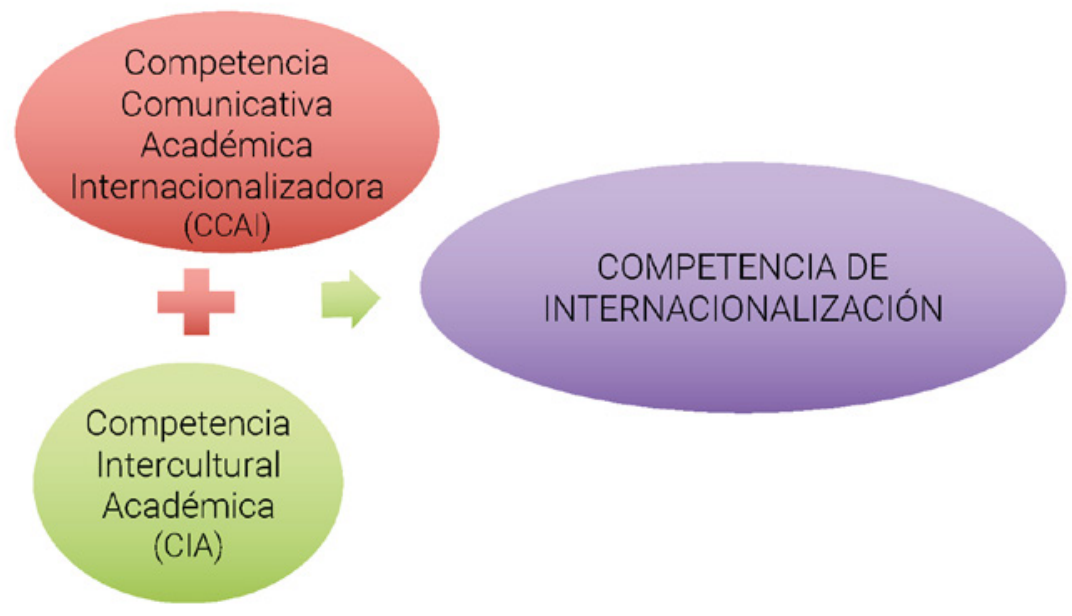

Figura 1. Modelo de competencia de internacionalización

Esta competencia interactúa con el resto de competencias docentes para lograr la dimensión internacional del profesorado universitario (Perrenoud, 2004; Rey, 2000). Se trata, por tanto, de una competencia transversal que requiere del resto de competencias docentes del profesor universitario para afrontar las situaciones de internacionalización con éxito.

\section{Conclusiones}

No cabe duda de que la internacionalización es una palabra clave en la agenda de las instituciones de Educación Superior españolas. Quizás se han hecho muchos esfuerzos a un nivel superficial para conseguir este objetivo con políticas de movilidad, de intercambio, de consolidación de redes, pero sin tener en cuenta si los agentes involucrados en ella están realmente preparados o no para afrontar este reto. En la mayoría de los documentos que hemos analizado encontramos referencias a las competencias de internacionalización que deben adquirir los alumnos universitarios españoles para hacer frente a un mercado laboral global, pero ¿y el profesorado? ¿Acaso no es la universidad su mercado laboral? ¿No se ha convertido ésta en un espacio de educación internacional? En este artículo hemos apostado porque la verdadera internacionalización vendrá de la mano de una plena transformación de la formación del profesorado 
universitario que lo capacite para desempeñar su trabajo a nivel internacional. Se le está demandando que establezca redes internacionales, que participe en grupos de trabajo internacional, que investigue en universidades extranjeras, pero ¿es éste competente para ello? No se trata de que el profesorado universitario no sepa hacer su trabajo, sino que las condiciones de juego han cambiado y esta transformación debe también reflejarse en sus competencias como docente universitario.

En este artículo hemos querido reivindicar el papel que la competencia de internacionalización del profesorado universitario juega sobre el desarrollo internacional de la Universidad española, así como el derecho del personal docente e investigador de estas instituciones a ser formado en ella. El desarrollo de esta competencia permitirá no solo la proyección exterior del docente, sino también dotar a su lugar de trabajo, es decir, a su institución de Educación Superior, de una verdadera dimensión internacional. Supone superar esa etapa fallida de la internacionalización de la universidad española, de acuerdo con Haug (2010), y conseguir que los campus tengan un verdadero tinte internacional.

Creemos que queda un largo camino por recorrer, pero el reconocimiento de esta nueva competencia en los decálogos de competencias del docente universitario será el impulso que la Universidad española necesita en esta carrera por la internacionalización.

\section{Referencias bibliográficas}

Aneas, A. (2003). Competencias interculturales transversales: un modelo para la detección de necesidades de formación (Tesis inédita de doctorado). Barcelona: Universidad de Barcelona. Recuperado de http://www.tdx.cat/bitstream/handle/10803/2343/0.PREVIO.pdf?sequence=1

Barrow, C., Didou, S., \& Mallea, J. (2003). Globalization, Trade Liberalization and Higher Education. Holanda: Kluwer.

Bennett, M. J. (1986). Hacia ethnorelativism: Un modelo de desarrollo de la sensibilidad intercultural. Orientación Cross-cultural: Nuevas conceptualizaciones y usos, 27-70.

Bergan, S., \& Rauhvargers, A. (2006). Council of Europe higher education series N4: "Recognition in the Bologna Process: policy development and the road to good practice". Bruselas: Servicio de Publicaciones del Consejo de Europa.

Bozu, Z., \& Imbernón, F. (2016). El presente y el futuro de la formación permanente docente del profesorado universitario. Educación y ciencia, 5(45), 94-105.

Comisión de Expertos para la Reforma del Sistema Universitario Español (2013). Informe "Propuestas para la reforma y mejora de la calidad y eficiencia del sistema universitario español". Recuperado de: http://www.mecd.gob.es/prensa-mecd/ actualidad/2013/02/20130215-comision-expertos.html

Comisión Europea (2017). Modernisation of Higher Education in Europe: Academic Staff - 2017. Luxemburgo: Oficina de Publicaciones de la Unión Europea. Recuperado de: https://webgate.ec.europa.eu/fpfis/mwikis/eurydice/images/3/3c/209_Academic_Staff_2017.pdf

Consejo de Europa (1999). Declaración de Bolonia: Declaración Conjunta de los Ministros Europeos de Educación en Bolonia, 19 de junio de 1999. Recuperado de: http:// 
www.mecd.gob.es/dctm/boloniaeees/documentos/02que/declaracionbolonia. pdf?documentId=0901e72b8004aa6a

De Ketele, J. M. (2003). La formación didáctica y pedagógica de los profesores universitarios: luces y sombras. Revista de Educación, 331, 143-169.

De Wit, H. (2017). Global: Internationalization of Higher Education: Nine Misconceptions. En Understanding Higher Education Internationalization (pp. 9-12). SensePublishers, Rotterdam.

De Wit, H. (2020). Internationalization of higher education. Journal of International Students, 10(1), I-IV.

Esteban, R. M., \& Menjívar, S. V. (2011). Una mirada internacional a las competencias docentes universitarias: investigación en primera persona: profesores y estudiantes. Madrid: Octaedro Editorial.

Fernández Cruz, M., \& Gijón, J. (2011). Nuevas políticas de profesionalización docente en la Educación Superior. Journal for Educators, Teachers and Trainers JETT, 2, 92-106. Recuperado de: http://www.ugr.es/ jett/pdf/vol02_10_jett_fernandezcruz_gijon-puerta.pdf

Gijón, J., \& Crisol, E. (2012). La internacionalización de la Educación Superior. El caso del Espacio Europeo de Educación Superior. Revista de Docencia Universitaria. REDU, Monográfico: Buenas prácticas docente en la enseñanza universitaria, 10(1), 389-414. Recuperado de: http://redaberta.usc.es/redu

Hammer, M. R. (1999). A measure of intercultural sensitivity: The Intercultural Development Inventory. The intercultural sourcebook, 2, 61-72.

Harris, S. (2008). La dimensión internacional de la universidad: entre el modelo económico y el cultural. ESE. Estudios sobre Educación, 15, 87-98.

Haug, G. (2010). La internacionalización de la Educación Superior: más allá de la movilidad europea. La cuestión Universitaria, 6, 21.

Hymes, D. (1972). On communicative competence. Sociolinguistics, 269-293.

Jato, E., Muñoz, M. A., \& García, B. (2014). Las necessidades formativas del professorado universitário: un análisis desde el programa de formación docente de la Universidad de Santiago de Compostela. REDU. Revista de Docencia Universitaria, 12(4), 203-229.

Kahn, H. E., \& Agnew, M. (2017). Global learning through difference: Considerations for teaching, learning, and the internationalization of higher education. Journal of Studies in International Education, 21(1), 52-64.

Knight, K. (1999). Internacionalización de la Educación Superior. En H. De Witt \& J. Knight (Eds.), Calidad e internacionalización en la Educación Superior. México DF: ANUIES.

Lasagabaster, D., Cots, J. M., \& Mancho-Bares, G. (2013). Teaching staff's views about the internationalisation of higher education: The case of two bilingual communities in Spain. Multilingual Journal of Cross-Cultural and Interlanguage Communication, 32(6), 751-778.

Lyotard, J. F. (1994). La condición postmoderna. Madrid: Cátedra.

Mas, O. (2011). El profesor Universitario: Sus competencias y formación. Profesorado. Revista de currículum y formación del profesorado, 15(3) 195-211. Recuperado de: http://www.ugr.es/ recfpro/rev153COL1.pdf 
Medina, A., \& Domínguez, M. C. (2006). Los procesos de observación del prácticum: análisis de las competencias. Revista Española de Pedagogía, 233, 69-104.

Medina, A., Domínguez, M. C., \& Ribeiro, F. (2011). Formación del Profesorado Universitario en las Competencias Docentes. Revista de Historia Educativa Latinoamericana, 13(7), 119-138.

Ministerio de Educación (2007). Ley Orgánica 4/2007, de 12 de abril, por la que se modifica la Ley Orgánica 6/2001, de 21 de diciembre, de Universidades (BOE 13/04/2007).

Monereo, C. (2013). La investigación en la formación del profesorado universitario: hacia una perspectiva integradora. Infancia y Aprendizaje, 36(3), 281-289.

Monereo, C., Sánchez-Busques, S., \& Suñé, N. (2012). La enseñanza auténtica de competencias profesionales. Un proyecto de aprendizaje recíproco instituto-universidad. Profesorado. Revista de currículum y formación del profesorado, 16(1), 79-101.

Montes, D. A., \& Suarez, C. I. (2016). La formación docente universitaria: claves formativas de universidades españolas. Revista Electrónica de Investigación Educativa, 18(3), 51-64. Recuperado de: http://redie.uabc.mx/redie/article/view/996

Morales, R., \& Cabrera, J. (2012). Las competencias docentes transversales. Método de selección MiZona-CDT. REDU. Revista Electrónica de Docencia Universitaria, 10(2), 75-101.

Ortega, V. (2002). Tendencias de la Educación Universitaria en el siglo XXI. Arbor. Ciencia, Pensamiento y Cultura, 681, 67-81.

Ortega y Gasset, J. (1930). Misión de la Universidad. Revista de Occidente.

Palma, M. (2011). Innovación y aprendizaje: Un nuevo modelo para la formación universitaria: ¿Por qué y para qué?. Arbor. Ciencia, Pensamiento y Cultura, 187(3), $77-$ 81.

Perrenoud, P. (2004). Diez nuevas competencias para enseñar. Barcelona: Graó.

Pricewaterhousecoopers S. L. (2013). Temas candentes de la universidad española 2013. Recuperado de: http://kc3.pwc.es/local/es/kc3/publicaciones.nsf/V1/7E7ABBBC81074258C1257B33005EA050/\$FILE/Temas-Candentes-Universidad.pdf

Rey, B. (2000). ¿Existen las competencias transversales?. Educar, 26, 9-17.

Ruíz, C., Mas, O., Tejada, J., \& Navío, A. (2008). Funciones y escenarios del profesorado universitario. Apuntes para la definición del perfil basado en competencias. Revista de la Educación Superior, 37(2), 115-132.

Tejada, J. (2002). La formación de formadores. Apuntes para una propuesta de plan de formación. Educar, 30, 91-118.

Tejada, J. (2005). El trabajo por competencias en el prácticum: cómo organizarlo y cómo evaluarlo. Revista electrónica de Investigación educativa, 7(2), 1-31. Recuperado de: http://redie.uabc.mx/vo7no2/contenido-tejada.html

Tejada, J. (2009). Competencias docentes. Profesorado. Revista de currículum y formación de profesorado, 13(2), 1-15. Recuperado de: http://www.ugr.es/ recfpro/ rev132COL2.pdf

Torres, A. D., Badillo, M., Valentín, N. O., \& Ramírez, E. T. (2015). Las competencias docentes: el desafío de la Educación Superior. Innovación Educativa, 14(6), 129-146.

Valcárcel, M. (2005). Diseño y validación de actividades de formación para profesores y gestores en el proceso de armonización europea en Educación Superior, (Estudio EA2004-0036 subvencionado por el Programa de Estudios y Análisis de la Direc- 
ción General de Universidades del Ministerio de Educación y Ciencia). Córdoba: Ediciones Gráficas Vistalegre.

Zabalza, M. A. (2002). La enseñanza universitaria. El escenario y sus protagonistas. Madrid: Narcea.

Zabalza, M. Á., \& Beraza, M. Á. Z. (2003). Competencias docentes del profesorado universitario: calidad y desarrollo profesional (Vol. 4). Narcea ediciones.

Zabalza, M. A. (2012). La universidad de las competencias. REDU. Revista de docencia universitaria, 10(2), 11-14. 